\title{
Anxiolytic Effects of Chewing Gum During Preoperative Fasting and Patient-centered Outcome in Female Patients Undergoing Elective Gynecologic Surgery: Randomized controlled study
}

\author{
Yu Jeong Bang \\ Samsung Medical Center \\ Jong-Hwan Lee \\ Samsung Medical Center \\ Chung Su Kim \\ Samsung Medical Center \\ Yoo-Young Lee \\ Samsung Medical Center \\ Jeong-Jin Min ( $\square$ mjj177@g.skku.edu ) \\ Samsung Medical Center
}

\section{Research Article}

Keywords: preoperative anxiety, APAIS, gum group, control group

Posted Date: September 17th, 2021

DOI: https://doi.org/10.21203/rs.3.rs-885349/v1

License: (c) (i) This work is licensed under a Creative Commons Attribution 4.0 International License.

Read Full License

Version of Record: A version of this preprint was published at Scientific Reports on March 9th, 2022. See the published version at https://doi.org/10.1038/s41598-022-07942-6. 


\section{Abstract}

Although previous studies reported that chewing gum during the preoperative fasting has the benefits of alleviating anxiety and dry mouth, preoperative chewing gum has yet to be accepted as a standard practice due to conventional anesthetic custom. Our study aimed to prospectively evaluate the effects of gum chewing on preoperative anxiety and patient's discomfort in female patients undergoing gynecologic surgery. Ninety-three patients were enrolled and randomized either into conventional fasting group (control group) or chewing gum with fasting group (gum group). The control group was instructed to fast from 3 p.m on the day before surgery. The gum group performed preoperative fasting in the same manner, but was encouraged to chew gum freely during the fasting period. The primary endpoints were the degree of preoperative anxiety and discomfort immediately before surgery. For the evaluation of preoperative anxiety, Amsterdam preoperative anxiety and information scale (APAIS) was used. Preoperative gastric fluid volume and acidity were also measured as the secondary outcomes. Preoperative anxiety using APAIS was significantly lower in the gum group compared to the control group (17.8 vs. $20.9, p=0.009$ ). The APAIS sub-score of anxiety and information desire domain were also lower in the gum group. However, there was no significant difference in the gastric fluid analysis between the groups. In the female patients for elective gynecologic surgery, chewing gum during the preoperative fasting period helped to alleviate preoperative anxiety without additional increase of pulmonary aspiration risks.

Trial registration: KCT0004422 (05/11/2019, https://cris.nih.go.kr; registration number)

\section{Introduction}

'Nil per $\mathrm{OS}^{\prime}$ (NPO) is a prerequisite for elective surgery to minimize the risk of pulmonary aspiration and associated complications ${ }^{1}$. Currently, most authoritative guidelines suggest relaxed fasting rule to prohibit solid food for 6 hours and clear liquids for 2 hours before elective surgery ${ }^{1-4}$. However, depending on the progress of surgical procedures in the operating room, the NPO period tends to be longer than expected, and this prolonged fasting may increase preoperative discomfort or anxiety ${ }^{5}$.

Chewing gum has the effect of stress reduction, amelioration of dry mouth, and promote gastrointestinal motility ${ }^{6-8}$. Because gum has been treated as solid food by anesthesiologists, many institutional NPO guidelines forbid chewing gum during preoperative fasting, and some institutions recommend it only in the postoperative period to improve bowel recovery ${ }^{9-13}$. However, in several previous studies, preoperative gum-chewing did not increase the gastric content volumes and the risk of pulmonary aspiration ${ }^{14-16}$. Based on this, some opinions have recently emerged to allow or recommend chewing gum during the preoperative fasting period focusing on the potential benefits of alleviating anxiety and dry mouth ${ }^{15,17}$.

Female patients, especially those undergoing gynecological surgery, are known to have increased preoperative anxiety compared to male patients or female patients undergoing other minor or general surgery ${ }^{18-21}$. Increased preoperative anxiety may adversely influence patient recovery and satisfaction, 
so it is necessary to address the preoperative anxiety in this patient population. However, most studies of preoperative chewing gum have focused on its effects on gastric volume and acidity, and clinical data regarding the patient-centered benefits of alleviating preoperative anxiety and discomfort are still insufficient.

In this randomized clinical study, we aimed to evaluate the effects of chewing gum on preoperative anxiety and discomfort in female patients undergoing elective gynecologic surgery.

\section{Methods}

Study design. This study is a single-center prospective randomized controlled study. The study was conducted in accordance with the principles of the Declaration of Helsinki and the International Conference on Harmonisation of Good Clinical Practice guidelines. The study protocol was approved by the institutional review board of Samsung Medical Center (IRB number: SMC 2019-05-168-001) and registered at CRIS (https://cris.nih.go.kr; registration number KCT0004422; 05/11/2019). We enrolled female patients scheduled for elective gynecologic laparoscopic surgery between August 2019 and June 2020. Patients were included if they were 19-70 years of age with American Society of Anesthesiologists physical status $\mathbb{\nabla}$ - II. Patients at increased risk for pulmonary aspiration were excluded. Exclusion criteria were as follows: emergency conditions, body mass ind//ex $(\mathrm{BMI})>30$, previous esophageal or gastric surgery, gastroesophageal reflux, gastrointestinal disorders (including gastritis, hiatal hernia, diabetic gastroparesis), ileus and current medication affect gastrointestinal motility ${ }^{40-42}$. Eligible patients were randomly assigned to either conventional fasting group (control group) or chewing gum with fasting group (gum group) at a ratio of 1:1, according to a randomization list generated by random permuted block design with a block size of two.

Intervention and anesthesia. The internal policy of gynecologic department of our hospital had stipulated the guidelines about preoperative fasting and bowel preparation as follows: All patients scheduled for gynecologic surgery were instructed to ban solid food from 3 p.m. the day before surgery and clear liquids from midnight before surgery according to protocol of the department of gynecology. In addition, $170 \mathrm{~mL}$ of Picosolution ${ }^{\circledR}$ (Pharmbio Korea Inc: Seoul, South Korea) was administered orally for bowel preparation at $5 \mathrm{pm}$ on the day before surgery. Our patients were hospitalized around $5 \mathrm{p} . \mathrm{m}$. the day before surgery and invited to participate in this study. After obtaining informed consent, patients were requested to rate their preoperative anxiety as a baseline examination, on a NRS (numeric rating scale) of 0 to 10 by a blinded investigator.

Patients in the control group were requested to follow the fasting guidelines without further treatment. Patients in the gum group were allowed to chew gums during the fasting period, following the fasting protocols described above. We distributed 12 pieces of sugarless xylitol gum (Xylichew: Hayden, Idaho, USA). We asked participants to chew gum during preoperative fasting period and to stop chewing gum from departure time for the operating room on the day of surgery. Participants were basically instructed to chew gum freely. It was recommended to chew gum at least one piece of gum more than 10 miniutes 
per hour, except for sleep time. All participants in chewing gum group were asked to log their chewing time. On the day of surgery, all patients were instructed to remove any gum from their mouths immediately prior to departure for the operating room, and complete questionnaire about the degree of discomfort associated with fasting and anxiety in the preoperative holding area with the help of attending residents or nurses in anesthesia team who were not aware of group allocation. In the operating theater, just before general anesthesia was induced, US assessment of gastric fluid volume was performed in both groups. All ultrasound (US) exams for estimating gastric volume were performed by independent blinded investigator (YJ, Bang), who was instructed and trained by an experienced radiologist.

Then, a standardized anesthesia protocol was used for all patients. The standard ASA monitoring including noninvasive blood pressure, EKG, pulse oximetry, and bispectral index (BIS) monitoring was applied. After denitrogenation with $80 \%$ oxygen, general anesthesia was induced with propofol and remifentanil using target-controlled infusion (Orchestra ${ }^{\circledR}$ Base Primea; Fresenius Kabi, Brezins, France), and intravenous rocuronium $0.8 \mathrm{mgkg}^{-1}$. Another investigator who was not aware of group allocation performed intubations and evaluated the degree of oral secretion during intubation. After tracheal intubation via video stylet, the ventilator was set with a tidal volume of $8 \mathrm{mLkg}^{-1}$ of ideal body weight and $\mathrm{FiO}_{2} 40 \%$. The respiratory rate and $\mathrm{I}: \mathrm{E}$ ratio were adjusted to maintain inspiratory peak pressure less than $30 \mathrm{cmH}_{2} \mathrm{O}$ and normocapnia. During the whole surgery, propofol and remifentanil effect site concentrations were adjusted to achieve BIS values of 40 to 50 and to maintain mean blood pressure and heart rate within $20 \%$ of pre-induction values. At the end of surgery, the neuromuscular block was reversed with pyridostigmine $\left(250 \mathrm{mcgkg}^{-1}\right)$ and glycopyrrolate $\left(10 \mathrm{mcgkg}^{-1}\right)$. After confirming that spontaneous breathing was sufficient and consciousness had returned, tracheal extubation was performed.

Postoperative recovery and bowel complication during the in-hospital period were evaluated and documented by independent gynecologists.

Postoperative management. The postoperative analgesia was standardized for all patients. If patients presented with breakthrough pain (NRS $\geq 4 / 10$ ), IV ibuprofen $400 \mathrm{mg}$ was administered. If this proved ineffective after 30 minutes IV pethidine $50 \mathrm{mg}$ was administered. Postoperative nausea and vomiting were treated with intravenous metoclopramide $10 \mathrm{mg}$ and ramosetron $0.3 \mathrm{mg}$. All patients resumed diet and ambulation as soon as possible after full recovery from anesthesia unless gastrointestinal symptoms were noted. Hospital discharge was determined by the surgery team.

Data collection and outcomes. Primary outcomes were preoperative anxiety and severity of symptoms related to preoperative fasting. The parameters used to assess discomfort were as follows: hunger, thirst, dry mouth, fatigue, headache, nausea using a single point NRS; $0=$ no suffer to $10=$ worst suffer imaginable ${ }^{43}$. We investigated preoperative anxiety using the Korean version of Amsterdam Preoperative Anxiety and Information Scale (APAIS) ${ }^{44,45}$. APAIS is a useful tool to evaluate preoperative stress and anxiety, which consists of an anxiety scale and a need for information scale. The scores on the anxiety of 
APAIS range from 4 (not anxious) to 20 (extremely anxious). For gum group only, the satisfaction with chewing gum during the preoperative fasting period was investigated.

Secondary outcomes included the amount of oral secretion at the timing of tracheal intubation, estimated gastric volume just prior to induction of anesthesia, gastric fluid acidity, recovery of bowel function, composite of postoperative bowel complication, QoR-15 score (Quality of Recovery - 15), and the length of hospital stay (LOS). The amount of oral secretion was graded depending on the degree of interference with intubation as follows; none (no saliva, thick tongue or mucosa causing the endotracheal tube stuck), mild (some saliva with good visual field), moderate (much saliva with limited field), severe (much saliva with blocked field, need for suction). For evaluation of recovery of bowel function, we collected data about time to flatus, time to sips of water, and time to first meal. We collected the data about postoperative bowel complication such as nausea, vomiting, abdominal pain, and abdominal distension. The severity of postoperative bowel complication was classified according to Clavien Dindo classification ${ }^{46}$. On the day after surgery, patients completed Korean version of QoR-15 questionnaire, which provides an extensive effective evaluation of quality of recovery after surgery and anesthesia ${ }^{47}$.

Acidity of gastric fluid analysis. ST probe 12Fr G type (SST12; S\&S med Inc; Anyang, South Korea) was inserted trans-orally to monitor the core body temperature. ST probe is a multi-orificed silicone tube with suction hole for gastric decompression. After induction of anesthesia, we measured the length from the mouth to the mandible angle, then from the mandible angle to the midpoint between xiphoid and the umbilicus. Then ST probe was advanced through oropharyngeal airway to the previously identified length and the end tip of the ST probe was placed in the stomach. Patients were placed in extreme head down position by surgeon's request so that gastric fluid could be drained via suction hole, naturally. If gastric fluid does not flow out by the end of surgery, the patient is tilted to the left to promote gastric fluid drainage. The acidity of the gastric fluid was analyzed with pH meter (PH60F Flat PH tester; Apera, Columbus, Ohio, USA) by blinded investigator.

Gastric ultrasonography. The aforementioned-investigator (YJ, Bang) performed US assessment using a portable US unit (Sonosite M- TURBO, Fujifilm Sonosite, Bothell, WA, USA) with a 2-5Hz convex probe. All patients underwent US exams of the epigastrium in the right lateral position. The gastric antrum was identified between the left lobe of the liver and the pancreas, at the level of the aorta, or the inferior vena cava. The cross-sectional area (CSA) was measured from serosa to serosa using the free tracing tool of the ultrasound machine. If the antrum had a perfect elliptical shape, CSA was calculated using the following formula: CSA $\left(\mathrm{cm}^{2}\right)=($ anteroposterior diameter $[\mathrm{cm}] \times$ craniocaudal diameter $[\mathrm{cm}] \times \pi) / 4$. Estimated gastric fluid volume (EGFV) was calculated using the following formula: EGFV $(\mathrm{mL})=27.0+$ $14.6 \times$ Right lateral CSA $-1.28 \times$ age $^{42}$.

Sample size calculation and statistical analysis. The standard deviation of the APAIS score derived from the existing literature is $3.2^{44}$. Assuming that the APAIS value of the gum group compared to the control group must decrease by 2 points to detect clinically meaningful differences, the necessary sample size 
was 42 participants for each group with a power of 0.8 and an a value of 0.05 . We decided that 47 patients in each group were to be enrolled to account for an expected $10 \%$ attrition rate, with a total of 94 .

All data were tested for normality by the Shapiro-Wilk test and were presented as means (standard deviations [SD]) or as medians (interquartile ranges [IQR]). Differences between groups were analyzed using the chi-square test or Fisher's exact test for categorical variables, and Student's t test or Wilcoxon's rank sum test for continuous variables as appropriate. Post hoc analyses were also performed. Statistical significance was defined by $p$ values $<0.05$. All analyses were performed using SAS version 9.4 (SAS Institute Inc, Cary, NC, USA).

\section{Results}

A total of 97 patients were screened for eligibility. After excluding 3 patients (BMI > 30), 94 patients were randomized either into the control group or gum group. One patient in the gum group was excluded due to unexpected conversion of surgical approach to open laparotomy. Consequently, 93 patients (47 patients in the control group and 46 patients in the gum group) completed the study and were analyzed (Fig. 1). The demographic and perioperative data are summarized in Table 1. Age, BMI, and ASA physical status did not differ between groups. No differences were observed between groups in terms of fasting time or intraoperative data. The median value of chewing time in the gum group was 110 minutes (57-180). 
Table 1

Patient characteristics and perioperative data.

\begin{tabular}{|c|c|c|}
\hline Parameter & $\begin{array}{l}\text { Control group } \\
(N=47)\end{array}$ & $\begin{array}{l}\text { Gum group } \\
(N=46)\end{array}$ \\
\hline Age (yr), mean (SD) & $42.9(10.2)$ & $43.8(10.9)$ \\
\hline Height (cm), mean (SD) & $159.2(5.0)$ & $160.3(6.0)$ \\
\hline Weight (kg), mean (SD) & $59.5(8.4)$ & $57.8(6.9)$ \\
\hline Body mass index $\left(\mathrm{kg} \cdot \mathrm{m}^{-2}\right)$, median (IQR) & $22.5[21.1-25.9]$ & $22.4[21.0-24.0]$ \\
\hline ASA physical status (l; II), $n /$ total $N(\%)$ & $\begin{array}{l}27 / 47(57 \%) ; 20 / 47 \\
(43 \%)\end{array}$ & $\begin{array}{l}27 / 46(59 \%) ; 19 / 46 \\
(41 \%)\end{array}$ \\
\hline \multicolumn{3}{|l|}{ Occupation, $n /$ total $N(\%)$} \\
\hline Housewife & $21 / 41(51 \%)$ & $21 / 42(50 \%)$ \\
\hline Clerical worker & $10 / 41(24 \%)$ & $9 / 42(21 \%)$ \\
\hline Service worker & $4 / 41(10 \%)$ & $2 / 42(5 \%)$ \\
\hline Professionals & $3 / 41(7 \%)$ & $6 / 42(14 \%)$ \\
\hline Student & $0 / 41(0.0 \%)$ & $2 / 42(5 \%)$ \\
\hline Retired or unemployed & $3 / 41(7 \%)$ & $2 / 42(5 \%)$ \\
\hline \multicolumn{3}{|l|}{ Marital status, $n /$ total $N(\%)$} \\
\hline Married & $36 / 47(77 \%)$ & $30 / 46(65 \%)$ \\
\hline Unmarried, divorced, or bereaved & $11 / 47(23 \%)$ & $16 / 46(35 \%)$ \\
\hline Malignancy, $n /$ total $N(\%)$ & $17 / 47$ (36\%) & $19 / 46(41 \%)$ \\
\hline \multicolumn{3}{|l|}{ History of previous surgery, $n /$ total $N(\%)$} \\
\hline 0 & $13 / 47(28 \%)$ & $12 / 46(26 \%)$ \\
\hline $1-2$ & $31 / 47(66 \%)$ & $28 / 46(61 \%)$ \\
\hline$\geq 3$ & $3 / 47(6 \%)$ & $6 / 46(13 \%)$ \\
\hline \multicolumn{3}{|l|}{$\begin{array}{l}\text { History of previous anesthesia, } n / \text { total } N \\
(\%)\end{array}$} \\
\hline General anesthesia & $22 / 47(47 \%)$ & $22 / 46(48 \%)$ \\
\hline Regional anesthesia & 10/47 (21\%) & $9 / 46(20 \%)$ \\
\hline
\end{tabular}




\begin{tabular}{|c|c|c|}
\hline Parameter & $\begin{array}{l}\text { Control group } \\
(N=47)\end{array}$ & $\begin{array}{l}\text { Gum group } \\
(N=46)\end{array}$ \\
\hline MAC & $2 / 47(4 \%)$ & $3 / 46(7 \%)$ \\
\hline Anxiety about preparing for surgery & $2[0-5]$ & $2[0-5]$ \\
\hline Fasting time (hr) & $19.7[18.0-21.6]$ & $21.7[18.3-24.1]$ \\
\hline \multicolumn{3}{|l|}{ Extents of Surgery, $n$ /total $N(\%)$} \\
\hline Ovarian cystectomy & $4 / 47(9 \%)$ & 9/46 (20\%) \\
\hline Salpingo- oophorectomy & $8 / 47(17 \%)$ & $9 / 46(20 \%)$ \\
\hline Myomectomy & $8 / 47(17 \%)$ & $7 / 46(15 \%)$ \\
\hline Hysterectomy & $24 / 47(51 \%)$ & $17 / 46(37 \%)$ \\
\hline Miscellaneous & $3 / 47(6 \%)$ & $4 / 46(9 \%)$ \\
\hline \multicolumn{3}{|l|}{ Surgical access, $n /$ total $N(\%)$} \\
\hline Single port laparoscopic Surgery & $7 / 47(15 \%)$ & $6 / 46(13 \%)$ \\
\hline Dual port laparoscopic Surgery & $14 / 47(30 \%)$ & $23 / 46(50 \%)$ \\
\hline Conventional laparoscopic Surgery & $15 / 47(32 \%)$ & $16 / 46(35 \%)$ \\
\hline Robot assisted laparoscopic surgery & $11 / 47(23 \%)$ & $1 / 46(2 \%)$ \\
\hline Anesthetic time (min), mean (SD) & $144.0(51.1)$ & $133.4(38.7)$ \\
\hline Crystalloid (mL), mean (SD) & $778.7(303.9)$ & $685.9(226.2)$ \\
\hline Estimated blood loss (mL), median (IQR) & $50[50-100]$ & $100[50-150]$ \\
\hline Urine output (mL), median (IQR) & $100[0-120]$ & $50[0-120]$ \\
\hline Opioid (MED), median (IQR) & $6.01[3.35-9.34]$ & $6.64[3.33-10.02]$ \\
\hline
\end{tabular}

The degree of anxiety assessed using APAIS is shown in Fig. 2. The mean value of APAIS and sub-scores of anxiety domain of APAIS were significantly lower in the gum group compared to the control group (20.9 [5.7] vs. 17.8 [5.5], $p=0.009$ and 14.1 [4.1] vs. 12.3 [3.8], $p=0.04$, respectively) (Table 2). The median value of information desire domain of APAIS shows significant different between groups (6.0 [4.0-7.0] vs. 7.0 [6.0-8.0], $p=0.002$ ). However, patient discomfort related to preoperative fasting such as hunger, thirst, dry mouth, fatigue, headache, and nausea did not differ between groups. There was no significant correlation between chewing time and preoperative discomfort and anxiety, but only weak inverse correlation with headache (Spearman correlation coefficient $=-0.034, p=0.022$ ). 
Table 2

Preoperative patient discomfort and gastric contents analysis

\begin{tabular}{|c|c|c|c|c|}
\hline Parameter & $\begin{array}{l}\text { Control } \\
\text { group } \\
(N=47)\end{array}$ & $\begin{array}{l}\text { Gum } \\
\text { group } \\
(N=46)\end{array}$ & $\begin{array}{l}\text { Difference in means } \\
(95 \% \mathrm{Cl})\end{array}$ & $\begin{array}{l}P \\
\text { value }\end{array}$ \\
\hline APAIS - total, mean (SD) & $20.9(5.7)$ & $17.8(5.5)$ & $3.1(0.8$ to 5.4$)$ & $0.009^{a}$ \\
\hline APAIS - anxiety, mean (SD) & $14.1(4.1)$ & $12.3(3.8)$ & 1.8 (0.1 to 3.4$)$ & $0.04^{a}$ \\
\hline \multirow[t]{2}{*}{$\begin{array}{l}\text { APAIS - information desire, median } \\
\text { (IQR) }\end{array}$} & \multirow[t]{2}{*}{$7[6-8]$} & \multirow[t]{2}{*}{$6[4-7]$} & $\begin{array}{l}\text { Difference in medians } \\
(95 \% \mathrm{Cl})\end{array}$ & \multirow[t]{2}{*}{$0.002^{b}$} \\
\hline & & & $1.0(-0.2$ to 2.2$)$ & \\
\hline \multicolumn{5}{|l|}{ Preoperative discomfort } \\
\hline Hunger, median (IQR) & $2[0-5]$ & $3[0-5]$ & $-1(-3.0$ to 1.0$)$ & $0.65^{\mathrm{b}}$ \\
\hline Thirst, median (IQR) & $4[0-5]$ & $3[1-5]$ & $1(-0.7$ to 2.7$)$ & $0.85^{\mathrm{b}}$ \\
\hline Dry mouth, median (IQR) & $4[1-5]$ & $3[1-5]$ & $1(-0.7$ to 2.7$)$ & $0.43^{b}$ \\
\hline Fatigue, median (IQR) & $3[0-6]$ & $3.5[1-5]$ & $0(-2.2$ to 2.2$)$ & $0.86^{\mathrm{b}}$ \\
\hline Headache, median (IQR) & $0[0-7]$ & $1[0-4]$ & $-1(-3.0$ to 1.0$)$ & $0.80^{\mathrm{b}}$ \\
\hline Nausea, median (IQR) & $0[0-2]$ & $0[0-1]$ & $0(-0.8$ to 0.8$)$ & $0.13^{\mathrm{b}}$ \\
\hline Oral secretion & & & $\begin{array}{l}\text { Absolute risk } \\
\text { difference }(95 \% \mathrm{Cl})\end{array}$ & $0.94^{c}$ \\
\hline None & 2 & 2 & $-0.1(-8.3$ to 8.2$)$ & \\
\hline Mild & 34 & 32 & $-1.5(-20.0$ to 16.9$)$ & \\
\hline Moderate & 10 & 10 & $3.8(-12.8$ to 20.5$)$ & \\
\hline Severe & 1 & 2 & $-2.2(-9.4$ to 5.0$)$ & \\
\hline
\end{tabular}

Patients' discomfort related to preoperative fasting was rated using a numeric rating scale (NRS), 0 to 10. Denominators that do not equal the sample sizes are due to missing data.

aStudent's $\mathrm{t}$ test.

${ }^{b}$ Wilcoxon rank sum test.

'Fisher's exact test.

APAIS, the Amsterdam preoperative anxiety and information scale. 


\begin{tabular}{|c|c|c|c|c|}
\hline Parameter & $\begin{array}{l}\text { Control } \\
\text { group } \\
(N=47)\end{array}$ & $\begin{array}{l}\text { Gum } \\
\text { group } \\
(N=46)\end{array}$ & $\begin{array}{l}\text { Difference in means } \\
(95 \% \mathrm{Cl})\end{array}$ & $\begin{array}{l}P \\
\text { value }\end{array}$ \\
\hline \multirow[t]{2}{*}{ Gastric pH, median (IQR) } & $\begin{array}{l}1.34 \\
{[0.42-} \\
2.78]\end{array}$ & $\begin{array}{l}1.45 \\
{[0.55-} \\
2.2]\end{array}$ & $-0.10(-1.10$ to 0.90$)$ & $0.95^{\mathrm{b}}$ \\
\hline & $n=37$ & $n=36$ & & \\
\hline \multirow[t]{2}{*}{$\begin{array}{l}\text { Estimated gastric fluid volume } \\
\left(\mathrm{ml} \cdot \mathrm{kg}^{-1}\right) \text {, median (IQR) }\end{array}$} & $\begin{array}{l}0.14[0- \\
0.58]\end{array}$ & $\begin{array}{l}0.24[0- \\
0.62]\end{array}$ & $-0.09(-0.35$ to 0.17$)$ & $0.70^{\mathrm{b}}$ \\
\hline & $n=45$ & $n=46$ & & \\
\hline \multicolumn{5}{|c|}{$\begin{array}{l}\text { Patients' discomfort related to preoperative fasting was rated using a numeric rating scale (NRS), } 0 \text { to } \\
\text { 10. Denominators that do not equal the sample sizes are due to missing data. }\end{array}$} \\
\hline \multicolumn{5}{|l|}{ aStudent's t test. } \\
\hline \multicolumn{5}{|l|}{ bWilcoxon rank sum test. } \\
\hline \multicolumn{5}{|l|}{ 'Fisher's exact test. } \\
\hline APAIS, the Amsterdam preopera & ety and it & ation sc & & \\
\hline
\end{tabular}

Regarding gastric fluid volume and acidity, there were no significant differences between the two groups (Table 2). The amounts of oral secretion graded at tracheal intubation also did not differ between groups.

Postoperative bowel recovery, bowel complication during the in-hospital period, and LOS did not show significant difference between groups (Table 3). However, QoR-15 score, representing the subjective recovery, was significantly higher in the chewing gum group. 
Table 3

Postoperative outcomes of bowel function recovery

\begin{tabular}{|c|c|c|c|c|}
\hline Parameter & $\begin{array}{l}\text { Control group } \\
(N=47)\end{array}$ & $\begin{array}{l}\text { Gum group } \\
(N=46)\end{array}$ & $\begin{array}{l}\text { Difference in medians } \\
(95 \% \mathrm{Cl})\end{array}$ & $\begin{array}{l}p \\
\text { value }\end{array}$ \\
\hline \multirow[t]{2}{*}{$\begin{array}{l}\text { Time to flatus (hr), median } \\
\text { (IQR) }\end{array}$} & $\begin{array}{l}26.4[15.2- \\
39.2]\end{array}$ & $\begin{array}{l}20.6[16.8- \\
38.9]\end{array}$ & $5.4(-6.2$ to 17.1$)$ & $0.53^{\mathrm{a}}$ \\
\hline & $n=34$ & $n=30$ & & \\
\hline $\begin{array}{l}\text { Time to sips (hr), median } \\
\text { (IQR) }\end{array}$ & $2.7[2.1-4.6]$ & $2.5[2.1-4.1]$ & $0.1(-0.8$ to 0.9$)$ & $0.65^{\mathrm{a}}$ \\
\hline $\begin{array}{l}\text { Resume feeding (hr), } \\
\text { median (IQR) }\end{array}$ & $\begin{array}{l}19.6[14.9- \\
21.7]\end{array}$ & $\begin{array}{l}17.0[12.7- \\
18.4]\end{array}$ & $2.5(0.0$ to 5.1$)$ & $0.11^{\mathrm{a}}$ \\
\hline $\begin{array}{l}\text { Time from sips to diet (hr), } \\
\text { median (IQR) }\end{array}$ & $15.0[9.0-19.0]$ & $\begin{array}{l}13.5[6.0- \\
16.0]\end{array}$ & $1.5(-2.1$ to 5.1$)$ & $0.12^{\mathrm{a}}$ \\
\hline \multirow[t]{2}{*}{$\begin{array}{l}\text { Total bowel complication, } \\
n / \text { total } N(\%)\end{array}$} & 32 /47 (68\%) & $23 / 46(50 \%)$ & $\begin{array}{l}\text { Absolute Risk } \\
\text { difference }(95 \% \mathrm{Cl})\end{array}$ & $0.08^{\mathrm{b}}$ \\
\hline & & & $18.1(-1.6$ to 37.7$)$ & \\
\hline Nausea & 26 & 20 & 11.8 (-8.3 to 32.0$)$ & $0.25^{\mathrm{b}}$ \\
\hline Vomiting & 3 & 6 & $-6.7(-18.6$ to 5.3$)$ & $0.32^{c}$ \\
\hline Postprandial pain & 8 & 3 & $10.5(-2.4$ to 23.4$)$ & $0.12^{\mathrm{b}}$ \\
\hline Abdominal distension & 0 & 2 & $-4.4(-10.2$ to 1.6$)$ & $0.24^{\mathrm{c}}$ \\
\hline \multicolumn{5}{|l|}{ Clavien Dindo classification } \\
\hline Class 1 & 31 & 22 & $1.2(-9.1$ to 11.5$)$ & $\begin{array}{l}> \\
0.999^{c}\end{array}$ \\
\hline Class 2 & 1 & 1 & $-1.2(-11.5$ to 9.1$)$ & \\
\hline \multirow[t]{2}{*}{ Rescue antiemetics } & $0[0-1]$ & $0[0-1]$ & $\begin{array}{l}\text { Difference in medians } \\
(95 \% \mathrm{Cl})\end{array}$ & $0.32^{\mathrm{a}}$ \\
\hline & & & $0.0(-0.5$ to 0.5$)$ & \\
\hline $\begin{array}{l}\text { Discharge delay, } n / \text { total } N \\
(\%)\end{array}$ & 4 /47 (9\%) & 2 /46 (4\%) & $4.2(-5.8$ to 14.1$)$ & $0.68^{\mathrm{c}}$ \\
\hline
\end{tabular}

Denominators that do not equal the sample sizes are due to missing data.

${ }^{a}$ Wilcoxon rank sum test.

${ }^{\mathrm{b}}$ Chi-squared test.

${ }^{\mathrm{C}}$ Fisher's exact test. 


\begin{tabular}{|lllll|}
\hline Parameter & $\begin{array}{l}\text { Control group } \\
(\boldsymbol{N = 4 7})\end{array}$ & $\begin{array}{l}\text { Gum group } \\
(\boldsymbol{N = 4 6 )}\end{array}$ & $\begin{array}{l}\text { Difference in medians } \\
(\mathbf{9 5 \%} \mathrm{Cl})\end{array}$ & $\begin{array}{l}\boldsymbol{p} \\
\text { value }\end{array}$ \\
\hline QoR - 15 score, mean (SD) & $96.8(27.7)$ & $116.0(21.0)$ & $\begin{array}{l}\text { Difference in means } \\
(95 \% \mathrm{Cl})\end{array}$ & $0.001^{\mathrm{a}}$ \\
& $n=37$ & $n=36$ & $\begin{array}{l}-19.2(-30.7 \text { to }-7.6) \\
\text { Difference in medians } \\
(95 \% \mathrm{Cl})\end{array}$ & $0.22^{\mathrm{a}}$ \\
\hline $\begin{array}{l}\text { Hospital stay (day), median } \\
\text { (IQR) }\end{array}$ & $2[2-3]$ & $2[1-3]$ & $0.0(-0.8$ to 0.8)
\end{tabular}

\section{Discussion}

In this randomized controlled study, chewing gum during preoperative fasting alleviated preoperative anxiety and promoted patient-reported quality of postoperative recovery, without increasing the risk of pulmonary aspiration in female patients undergoing gynecological surgery.

Chewing or sham feeding in stressful situations has been shown to decrease plasma cortisol levels and reduce mental stress, especially in acute stress ${ }^{22-24}$. In the current study, patients who chewed gum showed significantly lower anxiety levels at the preoperative holding area, consistent with those previous researches. Preoperative anxiety is influenced by multiple factors such as patient personality, past experiences, or education level $18,20,25$. Therefore, an individualized approach is needed to alleviate anxiety, which requires additional medical personnel or costs. However, preoperative gum chewing can be used easily as a strategy to relieve anxiety for almost all patients, without the consumption of expensive medical resources. In addition, the reduced immediate pre-operative anxiety appears to be associated with a higher patient-reported quality of recovery score evaluated by QoR - 15, which may be interpreted to indicate the patient's sense of well-being in the perioperative period.

The average level of preoperative anxiety in our study was higher than previously reported by other investigations. The mean value of total APAIS in previous studies varies from $8.31-14.50^{18,26-28}$. The participants in the present study showed mean APAIS value of $19.3(5.8)$ in this study. We consider the reasons for inconsistency between our findings as follows. In previous studies, women, gynecological surgery, and cancer surgery were cited as important factors for preoperative anxiety ${ }^{18,27}$. All participants of our study were female patients undergoing gynecological surgery, and $41 \%$ of them were already diagnosed as cancer or BRCA gene (+). Second, according to previous studies, anxiety increased as the 
operation approached ${ }^{29,30}$. In our study, anxiety was measured immediately before entering the operating room, whereas previous study investigated preoperative anxiety at outpatient clinics for pre-anesthetic evaluation a few days prior to surgery. Jiwanmall et al investigated the preoperative anxiety in patients on the day of surgery and reported that mean value in anxiety domain of APAIS was 13.8 (3.54) and mean value in information desire domain of APAIS was 7.17 (1.72), which was similar result with this study ${ }^{29}$. In addition, we consider that the excessively long fasting time also affected increasing preoperative anxiety ${ }^{5}$.

The major concerns related to chewing gum during preoperative fasting is possibly increased risk of pulmonary aspiration by increased gastric fluid volume and acidity ${ }^{15,16,31-34}$. In the present study, factors that could affect pulmonary aspiration risk such as saliva secretion, gastric fluid volume, and gastric fluid acidity remained unchanged after gum chewing. Gastric volume of up to $1.5 \mathrm{~mL} . \mathrm{kg}^{-1}$ is reported to be within the normal range in fasted adults ${ }^{35,36}$, and $97 \%$ of subjects in this study had a completely empty stomach $\left(\leq 1.5 \mathrm{ml} \mathrm{kg}^{-}{ }^{1}\right)$. The mean value of gastric volume was $0.4 \mathrm{ml} \mathrm{kg}^{-}{ }^{1}$ in both groups. In terms of gastric acidity, gastric fluid $\mathrm{pH}$ was not different between the groups.

An important precaution when patients are allowed to chew gum during the preoperative NPO period is to ensure that the patient removes gum from the mouth before induction of anesthesia. In our protocol, participants were instructed to dispose of gum before departure from the ward on the day of operation. There were no adverse events caused by chewing gum such as airway obstruction, but one patient chewed gum in the preoperative holding area. If patients chew gum during pre-anesthetic fasting, there should be a step to ensure that the gum has been removed before entering the operating room.

Our study has clinical implications in the following aspects. The data collection was prospective in nature and conducted by a clinician not involved in postoperative patient care. While previous studies have focused on the safety of chewing gum in preoperative fasting, the current study evaluated the effects of chewing gum in preoperative fasting on patient anxiety, discomfort, satisfaction, and postoperative recovery from various angles, confirming safety as well. In particular, it has the advantage of presenting the variables of postoperative recovery comprehensively, from patient subjective reports to objective indicators. Our study is meaningful in that the quality of recovery was evaluated both from the perspective of the patients and the medical staff.

Despite these strengths, this study has several limitations. The main limitation is the lack of blinding. Due to the nature of the study design, study participants were not blinded about their group allocation. Second, we did not measure the baseline APAIS score, which could be a potentially major confounding factor. Although APAIS is a simple questionnaire to evaluate preoperative anxiety, practical problems of time constraints made it difficult to acquire baseline APAIS score. Instead of APAIS, we investigated patients' anxiety with NRS as baseline anxiety. Although it cannot replace the baseline APAIS score, we believe it reflects the patient's anxiety level before surgery. Third, participants were limited to female adults with ASA physical status $\mathbb{\nabla}$ - II scheduled gynecologic laparoscopic surgery. It is difficult to generalize the results of the current study to pediatric, elderly, and specific disease cohorts. Although we 
verified that chewing gum has positive effects on preoperative anxiety and promotes postoperative recovery without further risk, future research is needed to investigate whether chewing gum is beneficial, especially for patients with increased pulmonary aspiration risk. Forth, in this study protocol, chewing gum was encouraged freely only prior to surgery without standardized instruction, so it is difficult to determine whether gum had the effect of promoting bowel recovery, which has been reported as an advantage of chewing gum after cesarean section, gynecologic surgery, and colorectal surgery ${ }^{37-39}$. There is a possibility that the potential benefits of chewing gum in preoperative fasting was attenuated because the preoperative NPO time was longer than necessary due to the internal policy of our hospital's gynecology department. Future research is needed on the integrative benefits of pre- and postoperative gum chewing in the perioperative period. Nevertheless, chewing gum in preoperative fasting had positive effects in reducing anxiety and promoting recovery in this study.

\section{Conclusion}

This randomized controlled trial in female patients undergoing elective gynecologic surgery demonstrated that chewing gum during the preoperative fasting period helped to alleviate preoperative anxiety without affecting gastric volume or $\mathrm{pH}$ values. Further studies are needed examining the effects of preoperative chewing gum on other patient populations in groups of different aged or in different perioperative conditions.

\section{Declarations}

\section{Author contributions}

J.M. designed and conceived the study, interpret data, and drafted the manuscript. Y.B. conceived the study, participated in study design, performed statistical analysis, and drafted the manuscript. J.L and C.K. acquired data, coordinated data collection, and helped to draft the manuscript. Y.L participated to design the study, conducted data collection, and substantially revised the draft. All authors read and approved the final manuscript.

\section{Funding}

None.

\section{Competing interests}

The authors declare no competing interests.

\section{References}

1. Practice Guidelines for Preoperative Fasting and the Use of Pharmacologic Agents to Reduce the Risk of Pulmonary Aspiration. Application to Healthy Patients Undergoing Elective Procedures: An 
Updated Report by the American Society of Anesthesiologists Task Force on Preoperative Fasting and the Use of Pharmacologic Agents to Reduce the Risk of Pulmonary Aspiration., 126, 376-393 https://doi.org/10.1097/aln.0000000000001452 (2017).

2. Dobson, G. et al. Guidelines to the Practice of Anesthesia - Revised Edition 2019. Can. J. Anaesth, 66, 75-108 https://doi.org/10.1007/s12630-018-1248-2 (2019).

3. Fawcett, W. J. \& Thomas, M. Pre-operative fasting in adults and children: clinical practice and guidelines. Anaesthesia, 74, 83-88 https://doi.org/10.1111/anae.14500 (2019).

4. Smith, I. et al. Perioperative fasting in adults and children: guidelines from the European Society of Anaesthesiology. Eur. J. Anaesthesiol, 28, 556-569 https://doi.org/10.1097/EJA.0b013e3283495ba1 (2011).

5. Power, S. et al. Reducing preoperative fasting in elective adult surgical patients: a case-control study. Ir. J. Med. Sci, 181, 99-104 https://doi.org/10.1007/s11845-011-0765-6 (2012).

6. Apostolopoulos, P. et al. Clinical trial: effectiveness of chewing-gum in accelerating capsule endoscopy transit time-a prospective randomized, controlled pilot study. Aliment. Pharmacol. Ther, 28, 405-411 https://doi.org/10.1111/j.1365-2036.2008.03762.x (2008).

7. Noble, E. J., Harris, R., Hosie, K. B., Thomas, S. \& Lewis, S. J. Gum chewing reduces postoperative ileus? A systematic review and meta-analysis. Int. J. Surg, 7, 100-105 https://doi.org/10.1016/j.ijsu.2009.01.006 (2009).

8. Yang, P., Long, W. J. \& Wei, L. Chewing Xylitol Gum could Accelerate Bowel motility Recovery after Elective Open Proctectomy for Rectal Cancer. Rev. Invest. Clin, 70, 53-58 https://doi.org/10.24875/ric.18002428 (2018).

9. Nelson, G. et al. Guidelines for perioperative care in gynecologic/oncology: Enhanced Recovery After Surgery (ERAS) Society recommendations-2019 update. Int. J. Gynecol. Cancer, 29, 651-668 https://doi.org/10.1136/ijgc-2019-000356 (2019).

10. Darvall, J. N., Handscombe, M. \& Leslie, K. Chewing gum for the treatment of postoperative nausea and vomiting: a pilot randomized controlled trial. Br. J. Anaesth, 118, 83-89 https://doi.org/10.1093/bja/aew375 (2017).

11. Altraigey, A. et al. The effect of gum chewing on the return of bowel motility after planned cesarean delivery: a randomized controlled trial. J. Matern. Fetal Neonatal Med, 1-8 https://doi.org/10.1080/14767058.2018.1526913 (2018).

12. Lim, P. et al. Sham feeding with chewing gum after elective colorectal resectional surgery: a randomized clinical trial. Ann. Surg, 257, 1016-1024 https://doi.org/10.1097/SLA.0b013e318286504a (2013).

13. Xu, C., Peng, J., Liu, S. \& Qi, D. Y. Effect of chewing gum on gastrointestinal function after gynecological surgery: A systematic literature review and meta-analysis. J. Obstet. Gynaecol. Res, 44, 936-943 https://doi.org/10.1111/jog.13602 (2018).

14. Bouvet, L., Loubradou, E., Desgranges, F. P. \& Chassard, D. Effect of gum chewing on gastric volume and emptying: a prospective randomized crossover study. Br. J. Anaesth, 119, 928-933 
https://doi.org/10.1093/bja/aex270 (2017).

15. Poulton, T. J. Gum chewing during pre-anesthetic fasting. Paediatr. Anaesth, 22, 288-296 https://doi.org/10.1111/j.1460-9592.2011.03751.x (2012).

16. Schoenfelder, R. C., Ponnamma, C. M., Freyle, D., Wang, S. M. \& Kain, Z. N. Residual gastric fluid volume and chewing gum before surgery. Anesth. Analg, 102, 415-417 https://doi.org/10.1213/01.ane.0000189218.07293.6e (2006).

17. Garcia, A. K. A. et al. Menthol chewing gum on preoperative thirst management: randomized clinical trial. Rev. Lat. Am. Enfermagem, 27, e3180 https://doi.org/10.1590/1518-8345.3070.3180 (2019).

18. Eberhart, L. et al. Preoperative anxiety in adults - a cross-sectional study on specific fears and risk factors. BMC Psychiatry, 20, 140 https://doi.org/10.1186/s12888-020-02552-w (2020).

19. Jarmoszewicz, K., Nowicka-Sauer, K., Zemła, A. \& Beta, S. Factors Associated with High Preoperative Anxiety: Results from Cluster Analysis. World J. Surg, 44, 2162-2169 https://doi.org/10.1007/s00268-020-05453-x (2020).

20. Kain, Z. N., Sevarino, F., Alexander, G. M., Pincus, S. \& Mayes, L. C. Preoperative anxiety and postoperative pain in women undergoing hysterectomy. A repeated-measures design. J. Psychosom. Res, 49, 417-422 https://doi.org/10.1016/s0022-3999(00)00189-6 (2000).

21. Kindler, C. H., Harms, C., Amsler, F., Ihde-Scholl, T. \& Scheidegger, D. The visual analog scale allows effective measurement of preoperative anxiety and detection of patients' anesthetic concerns. Anesth. Analg, 90, 706-712 https://doi.org/10.1097/00000539-200003000-00036 (2000).

22. Kubo, K. Y., linuma, M. \& Chen, H. Mastication as a Stress-Coping Behavior. Biomed. Res. Int. 2015, 876409, http://doi.org/10.1155/2015/876409 (2015).

23. Sketchley-Kaye, K., Jenks, R., Miles, C. \& Johnson, A. J. Chewing gum modifies state anxiety and alertness under conditions of social stress. Nutr. Neurosci, 14, 237-242 https://doi.org/10.1179/1476830511y.0000000017 (2011).

24. Smith, A. P. Chewing gum and stress reduction. J. Clin. Transl. Res, 2, 52-54 (2016).

25. Jawaid, M., Mushtaq, A., Mukhtar, S. \& Khan, Z. Preoperative anxiety before elective surgery. Neurosciences (Riyadh), 12, 145-148 (2007).

26. Aust, H. et al. A cross-sectional study on preoperative anxiety in adults. J. Psychosom. Res, 111, 133-139 https://doi.org/10.1016/j.jpsychores.2018.05.012 (2018).

27. Celik, F. \& Edipoglu, I. S. Evaluation of preoperative anxiety and fear of anesthesia using APAIS score. Eur. J. Med. Res, 23, 41 https://doi.org/10.1186/s40001-018-0339-4 (2018).

28. Goebel, S. \& Mehdorn, H. M. Assessment of preoperative anxiety in neurosurgical patients: Comparison of widely used measures and recommendations for clinic and research. Clin. Neurol. Neurosurg, 172, 62-68 https://doi.org/10.1016/j.clineuro.2018.06.036 (2018).

29. Jiwanmall, M. et al. Preoperative Anxiety in Adult Patients Undergoing Day Care Surgery: Prevalence and Associated Factors. Indian J. Psychol. Med, 42, 87-92 https://doi.org/10.4103/ijpsym.ljpsym_180_19 (2020). 
30. Kumar, A., Dubey, P. K. \& Ranjan, A. Assessment of Anxiety in Surgical Patients: An Observational Study. Anesth. Essays Res, 13, 503-508 https://doi.org/10.4103/aer.AER_59_19 (2019).

31. Shanmugam, S., Goulding, G., Gibbs, N. M., Taraporewalla, K. \& Culwick, M. Chewing gum in the preoperative fasting period: an analysis of de-identified incidents reported to webAIRS. Anaesth. Intensive Care, 44, 281-284 https://doi.org/10.1177/0310057x1604400216 (2016).

32. Dubin, S. A., Jense, H. G., McCranie, J. M. \& Zubar, V. Sugarless gum chewing before surgery does not increase gastric fluid volume or acidity. Can. J. Anaesth, 41, 603-606 https://doi.org/10.1007/bf03010000 (1994).

33. Ouanes, J. P. et al. The role of perioperative chewing gum on gastric fluid volume and gastric $\mathrm{pH}$ : a meta-analysis. J. Clin. Anesth, 27, 146-152 https://doi.org/10.1016/j.jclinane.2014.07.005 (2015).

34. Soreide, E., Holst-Larsen, H., Veel, T. \& Steen, P. A. The effects of chewing gum on gastric content prior to induction of general anesthesia. Anesth. Analg, 80, 985-989 (1995).

35. Van de Putte, P. \& Perlas, A. The link between gastric volume and aspiration risk. search of the Holy Grail? Anaesthesia, 73, 274-279 https://doi.org/10.1111/anae.14164 (2018).

36. Roberts, R. B. \& Shirley, M. A. Reducing the risk of acid aspiration during cesarean section. Anesth. Analg, 53, 859-868 https://doi.org/10.1213/00000539-197453060-00010 (1974).

37. Altraigey, A. et al. The effect of gum chewing on the return of bowel motility after planned cesarean delivery: a randomized controlled trial. J. Matern. Fetal Neonatal Med, 33, 1670-1677 https://doi.org/10.1080/14767058.2018.1526913 (2020).

38. Chan, M. K. \& Law, W. L. Use of chewing gum in reducing postoperative ileus after elective colorectal resection: a systematic review. Dis. Colon Rectum, 50, 2149-2157 https://doi.org/10.1007/s10350007-9039-9 (2007).

39. Turkay, Ã., Yavuz, A., Hortu, Ä., Terzi, H. \& Kale, A. The impact of chewing gum on postoperative bowel activity and postoperative pain after total laparoscopic hysterectomy. J. Obstet. Gynaecol, 40, 705709 https://doi.org/10.1080/01443615.2019.1652891 (2020).

40. Birenbaum, A. et al. Effect of Cricoid Pressure Compared With a Sham Procedure in the Rapid Sequence Induction of Anesthesia: The IRIS Randomized Clinical Trial. JAMA Surg, 154, 9-17 https://doi.org/10.1001/jamasurg.2018.3577 (2019).

41. Song, I. K. et al. Ultrasound assessment of gastric volume in children after drinking carbohydratecontaining fluids. Br. J. Anaesth, 116, 513-517 https://doi.org/10.1093/bja/aew031 (2016).

42. Perlas, A. et al. Validation of a mathematical model for ultrasound assessment of gastric volume by gastroscopic examination. Anesth. Analg, 116, 357-363 https://doi.org/10.1213/ANE.0b013e318274fc19 (2013).

43. Doo, A. R., Hwang, H., Ki, M. J., Lee, J. R. \& Kim, D. C. Effects of preoperative oral carbohydrate administration on patient well-being and satisfaction in thyroid surgery. Korean J. Anesthesiol, 71, 394-400 https://doi.org/10.4097/kja.d.18.27143 (2018).

44. Moerman, N., van Dam, F. S., Muller, M. J. \& Oosting, H. The Amsterdam Preoperative Anxiety and Information Scale (APAIS). Anesth. Analg, 82, 445-451 (1996). 
45. Shin, W. J. et al. The Validity of Amsterdam Preoperative Anxiety Information Scale in the Assessment of the Preoperative Anxiety - Compared with hospital anxiety depression scale and visual analogue scale. Korean J. Anesthesiol, 37, 179-187 https://doi.org/10.4097/kjae.1999.37.2.179 (1999).

46. Dindo, D., Demartines, N. \& Clavien, P. A. Classification of surgical complications: a new proposal with evaluation in a cohort of 6336 patients and results of a survey. Ann. Surg, 240, 205-213 https://doi.org/10.1097/01.sla.0000133083.54934.ae (2004).

47. Kleif, J., Waage, J., Christensen, K. B. \& Gögenur, I. Systematic review of the QoR-15 score, a patientreported outcome measure measuring quality of recovery after surgery and anaesthesia. $\mathrm{Br}$. J. Anaesth, 120, 28-36 https://doi.org/10.1016/j.bja.2017.11.013 (2018).

\section{Figures}

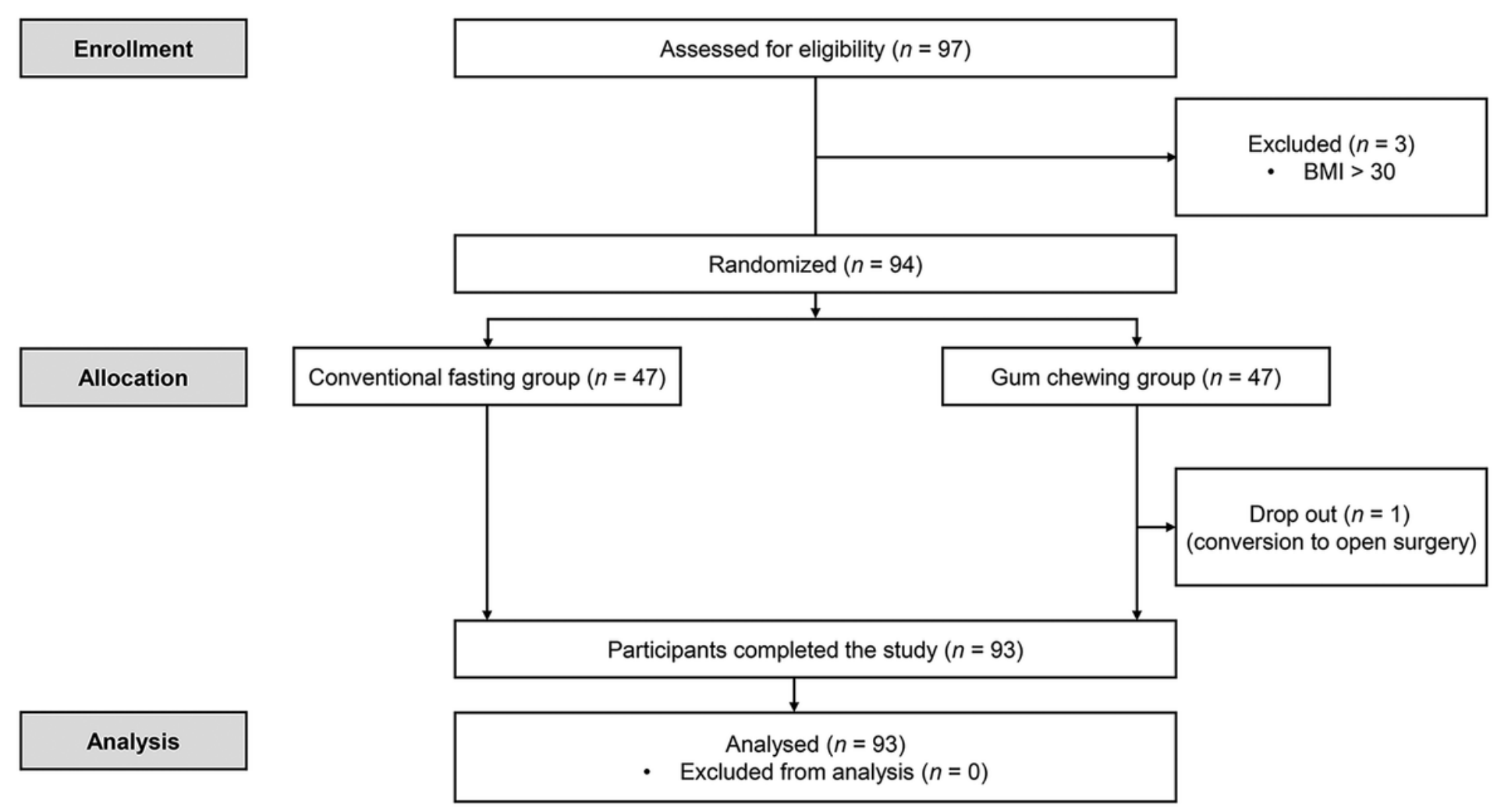

\section{Figure 1}

CONSORT flow diagram of patients included in the study. The conventional fasting group comprised patients who followed conventional preoperative fasting guidelines of ASA. The chewing gum group comprised patients who allowed to chew gum freely during the preoperative fasting period. ASA, American Society of Anesthesiologists. CONSORT, Consolidated Standards of Reporting Trials. 


\section{APAIS score}

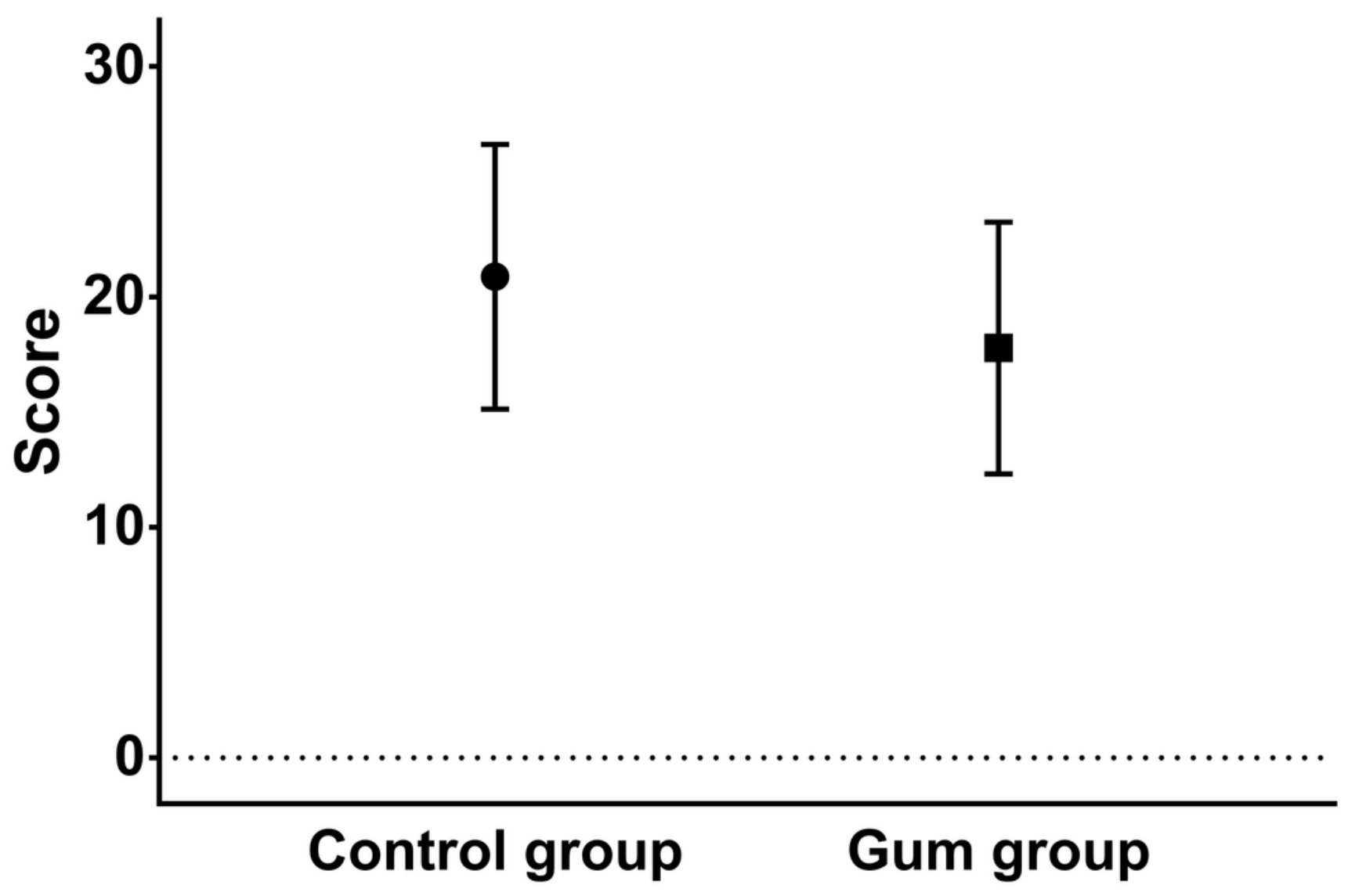

Figure 2

Comparison of patient anxiety scores using APAIS (the Amsterdam Preoperative anxiety and information Scale) before surgery. Data were analyzed using student's $t$ test. 